\title{
Solving the Transient Cost-Related Optimization Problem for Copper Flash Smelting Process with Legendre Pseudospectral Method
}

\author{
Jian-Hua Liu, Wei-Hua Gui, Yong-Fang Xie and Zhao-Hui Jiang* \\ School of Information Science \& Engineering, Central South University, Changsha, 410083, P. R. China
}

In the present contribution, a scheme to solve transient cost-related optimization problem of dynamic transient procedure for copper flash smelting process is investigated. Taking the actual copper flash smelting process at a Smelter in China as the research object, the transient costrelated optimization problem is presented by integrating transient time, transient resources consumption and the state fluctuation constraint. Then, it was considered as the operational parameters trajectories optimization problem that generates minimum-time and minimum-resources consumption during dynamic transient procedure. The dynamic relationship and the working state fluctuation are constructed in the form of constraints in the resulting optimization problem formulation. The Legendre Pseudospectral method is used to solve the constrained, nonlinear optimization problem. The results of numerical experiments of dynamic transient procedure for copper flash smelting process are given to illustrate the proposed scheme. [doi:10.2320/matertrans.M2012350]

(Received October 15, 2012; Accepted December 18, 2012; Published February 1, 2013)

Keywords: transient cost-related optimization, dynamic transient procedure, Legendre pseudospectral method, copper flash smelting process

\section{Introduction}

The pyrometallurgical flash smelting process is the main production method employed for extracting copper from its sulphide ores, ${ }^{1)}$ and it is widely used throughout the world for copper production, accounting for about $50 \%$ of global capacity for primary copper production. ${ }^{2,3)}$ However, along with the increase of the yield and the progressively depleting of the clean deposit sources, the copper concentrates involving copper are becoming more and more complex and low grade, ${ }^{4,5}$ which makes it difficult to maintain high performance level that used to meet the desired production indicator, resulting in great resources losses. ${ }^{6}$ In addition, the increasing energy demand from emerging economies versus the day-by-day decreasing storages of energy resource is implications that operator cannot any further disregard..$^{7-9)}$

The resources losses of copper concentrate may be attributed to two main factors: (i) probably for the reason of improper setting for operational parameters, the real-time running conditions deviate from the expectation, resulting in the insufficient extraction of copper concentrate resources; (ii) excessive time and/or copper concentrates resources may be consumed for dynamic transient procedure to change working conditions from real-time state to expectation, resulting in unnecessary waste of time and/or copper concentrate resources. Moreover, due to the complex about copper concentrate consisted of several separate streams, the dynamic transient procedure occurs frequently. For the former, the major concern is the extracted efficiency of copper concentrate resources for steady state process; the consumptions of time and resources of the dynamic transient procedure are mainly discussed for the later. Generally, the former problem may be solved by using model-based optimization method. ${ }^{10,11)}$ Many research studies have been carried out on it, Pradenas et al. apply a scheduling program to optimize the smelter production based on the finite-state machine model. ${ }^{12)}$ Model predictive control also has been proposed to reduce production costs by taking into account

*Corresponding author, E-mail: jzh0903@csu.edu.cn the cost of energy, raw material or manpower. ${ }^{13)}$ The industrial applications of research studies play an important role in improving the production yield and quality for copper smelting plant. However, most of these studies developed have been applied only for steady-state process to optimize the operational parameters. ${ }^{14-16)}$ It is difficult to optimization the dynamic transient procedure by using the steady-state model-based optimization methods due to the following hurdles: (i) the aim of transient cost-related optimization scheme is to change working conditions from real-time state to expectation, which is called dynamic transient procedure, the steady model used to describe the complex dynamic procedure have limited the validity; (ii) key state variables (such as matte grade) are measured intermittently with sampling intervals of approximately $1 \mathrm{~h},{ }^{14)}$ which makes it difficult to investigate the useful information (such as transient time, transient resources consumption, working state fluctuation, and so on) between intermittent time. In addition, little of the methods developed are perfect and all are far from ready to be used for dynamic transient procedure optimization. Thus, more researches will be regent required to solve the transient cost-related optimization problem of dynamic transient procedure for copper flash smelting process. Consequently, investigating a solving method for transient cost-related optimization problem for dynamic transient procedure is our thrust in this paper.

The main advantages of the present scheme are that: (i) the transient cost-related optimization problem, which is used to ensure the dynamic transient procedure optimization for copper flash smelting process, is presented a prior. (ii) with the optimization scheme proposed in this paper, the optimal operational parameters adjustment trajectories can be depicted detailed, this is useful in providing guidance for the adjustment of operational parameters. Meanwhile, the transient time and transient resources consumption during dynamic transient procedure also can be evaluated approximated; (iii) the impact degrees of different operational parameters adjustment on transient procedure of state parameters are different, which can be reflected by analyzing adjustment trajectories of operational parameters. 
In the remainder of the paper, combined with the actual copper flash smelting process at a Smelter in China, the transient cost-related optimization problem is presented and described as an operational parameters trajectories optimization problem, and the corresponding solving scheme is also investigated in Section 2. Section 3 briefly describes the construction of dynamic model, and the Legendre Pseudospectral method also be discussed in the section. The results and discussions are given in Section 4. Section 5 is the conclusion of this paper.

\section{Scheduling Problem}

\subsection{Process description}

Outokumpu flash smelter at a Smelter in China is pyrometallurgical equipment for smelting copper sulphide concentrates. The copper flash smelting process essentially consists of dispersing and oxidizing a dry concentrate in an oxygen-enriched air stream. ${ }^{17)}$ Both flows enter through a set of chambers and ducts into the concentrate burner which disperses the gas/solid mixture into a vertical reaction shaft. When the suspension leaves the reaction shaft, the reacted molten concentrate particles and inert flux particles are separated from the gas stream and hit towards molten slag and matte. In the molten bath, the reactions are completed basically and matte and slag will be settled at the settler of the furnace as respective layers due to their different density - a lighter slag phase rich in iron and a heavier matte phase rich in copper. ${ }^{18)}$

As a rule, the main variables of the copper flash smelting process are composition of the copper concentrate (mass fraction of $\mathrm{Cu}, \mathrm{Fe}, \mathrm{S}, \mathrm{SiO}_{2}$, et al., \%), the composition of the matte produced (mass fraction of $\mathrm{Cu}, \%$ ), composition of the slag produced (mass fraction of $\mathrm{Cu}, \mathrm{Fe}$ and $\mathrm{SiO}_{2}, \%$ ), and the matte temperatures $(\mathrm{K})$. Of these, the matte grade, which is used as target indicator practically, is one of the comprehensive indexes in the copper flash smelting process when the amount of the treated material is invariable for the copper flash smelting process. The stable matte grade is important to the smelting, converting and the production of sulfuric acid. ${ }^{19)}$ In practice, the feed flow of copper concentrate is usually calculated and fixed in advance by a software program based on mass and energy balances considering desired matte grade. So the controlling strategy for copper flash smelting process can be described: Based on the unchanged flow of feed, the optimization running of copper flash smelting process is mainly handled by adjusting the flow of oxygen-rich $\left(\mathrm{m}^{3} / \mathrm{h}\right)$ and the flow of air $\left(\mathrm{m}^{3} / \mathrm{h}\right)$, which are known as operational parameters. ${ }^{11)}$ Thus, we define the matte grade as state parameter that is to monitor running conditions of copper flash smelting process in this paper, the flow of oxygen-rich and air are defined as operational parameters. Also, the composition characteristics of copper concentrate feed are described as the condition parameters and assumed to be fixed during dynamic transient procedure.

\subsection{Problem description}

According to the flash smelting controlling strategy described previously, the smelting controlling strategy can be considered to chare dried copper concentrate to the furnace at a prescribed rate and to bass all the controls of oxygen-rich and air flow on this rate, and the aim of controlling is to change the working condition from real-time state to expectation. Practically, we always hope that the dynamic transient procedure can be completed as quickly as possible with consuming resources as few as possible. Since the dynamic transient procedure is mainly completed by the adjustment of operational parameters, the performance index of dynamic transient procedure is determined by the adjustment trajectories of operational parameters. However, which kind of adjustment trajectories of operational parameters should be implemented to ensure the dynamic transient procedure optimization? This is our major concerned problem in the paper. From the analysis above, the transient cost-related optimization problem also can be described as the following operational parameters trajectories optimization problem: Find the optimal operational parameters adjustment trajectories and the corresponding state transient procedure that minimize the following performance index function,

$$
\begin{aligned}
& \min J=G+\lambda \int_{t_{0}}^{t_{\mathrm{f}}} F \mathrm{~d} t \\
& \text { s.t. }\left\{\begin{array}{l}
x^{\min } \leq x(t) \leq x^{\max } \\
\frac{\mathrm{d} x(t)}{\mathrm{d} t}=f(x(t), I(t), u(t)) \\
u_{i}^{\min } \leq u_{i}(t) \leq u_{i}^{\max } \\
i=1,2
\end{array}\right.
\end{aligned}
$$

where, $J$ is the transient cost-related function constructed by integrating transient time and transient resources consumption; $G$ and $F$ are endpoint cost and running cost, respectively, and expressed by $G=t_{\mathrm{f}}-t_{0} ; t_{0}, t_{\mathrm{f}}$ are the start time and endpoint time for dynamic transient procedure; $F=\mu u_{1}(t)+(1-\mu) u_{2}(t), u_{1}(t)$ and $u_{2}(t)$ are the flow of oxygen-rich and air during dynamic transient procedure, respectively; $\lambda$ is the consumption coefficient of resources, and $\mu$ is the coefficient represented for the amount of oxygen-rich compared with all amount resources during dynamic transient procedure. $x(t)$ is the matte grade at time $t$, $x^{\mathrm{min}}$ and $x^{\mathrm{max}}$ are the allowed minimum and maximum values of matte grade with considering the downstream converters process. $u_{1}(t)$ and $u_{2}(t)$ represented for the flow of oxygenrich and air at time $t$, respectively. $u_{1}^{\min }, u_{i}^{\max }, u_{2}^{\min }$ and $u_{2}^{\max }$ are the minimum and maximum production capacity of flow of oxygen-rich and air, respectively.

In addition, the dramatic fluctuation of state parameter (matte grade) is harmful to production process, especially for the case near by the steady-state point. In order to avoid it, by using limiting the substantial adjustment for operational parameters, the following constraint is employed,

$$
\sigma_{i} \frac{\left\|u_{i}(t)-u_{i}^{*}\right\|}{u_{i}^{*}} \leq \operatorname{arctanh}\left(\frac{\left\|x(t)-x^{*}\right\|}{x^{*}}\right)
$$

In eq. (2), $x^{*}$ is the expectative state parameter represented for matte grade, and it is also the endpoint state of dynamic transient procedure, which is set up based on the real-time condition parameters such as the mass fraction of $\mathrm{Cu}, \mathrm{Fe}, \mathrm{S}$, $\mathrm{SiO}_{2}$ of the copper concentrate, et al.; $u_{i}^{*}(i=1,2)$ is the optimized operational parameters represented for the flow of oxygen-rich and air, respectively, by using model-based 
optimization methods. ${ }^{11,16)} \sigma_{i}(i=1,2)$ is the influence coefficient of the oxygen-rich and air flow on the fluctuation of state parameter, respectively, and $\sigma_{1}+\sigma_{2}=1$. The use of constraint eq. (2) is to ensure that the fluctuation of state parameter (matte grade) can be adjusted automatically according to the deviation of real-time state from expectation. It can be seen from eq. (2) that the operational parameters will be limited in a small adjustment domain when the real-state nears by the expectation, which is useful for the smoothness of dynamic transient procedure.

\subsection{Solving method investigation}

As mentioned previously, the transient cost-related optimization problem is described as an operational parameters trajectories optimization problem eqs. (1) and (2), where subject to equality and inequality hybrid constraints. The Pseudospectral (PS) methods are powerful tools for numerical solutions of integral and differential equations. ${ }^{20,21)}$ In this methods, polynomial approximations of the state and control variables are considered where Lagrange polynomial are the trial functions and the unknown coefficients are the values of the state and control variables at the LegendreGauss-Lobatto (LGL) point. ${ }^{22,23)}$ It is well known that this choice of collocation points yields superior results for interpolation of functions to the ones obtained from equidistant points. By using the properties of the Lagrange polynomials, the state differential constraints are imposed by evaluating the functions at the LGL points and using a derivative matrix that is obtained by taking the analytic derivative of the interpolating polynomials and evaluating them at the LGL points. The integral cost function can also be discretized by Gauss-Lobatto quadrature rules, which provide highly accurate results for approximating integrals. ${ }^{24,25)}$ The optimization problem is thereby converted into a nonlinear programming problem (NLP), ${ }^{26,27)}$ which can be solved by a well-developed parameter optimization algorithm. Thus, the integral and differential equations in eq. (1) can be discretized with PS methods to a NLP and solved by a well-developed parameter optimization algorithm. In addition, due to the fact that the fluctuation constraint eq. (2) is developed according to the deviation of real time operational parameter from the optimal operational parameter related to the expectation, the optimal operational parameter should be acquired in advance. So, the modelbased optimization methods still also are employed to acquire the final operational parameters, ${ }^{11,16)} u_{i}^{*}(i=1,2)$, namely the optimal operational parameters at the endpoint for the dynamic transient procedure.

In summary, the solving scheme for transient cost-related optimization problem eqs. (1) and (2) is summarized as: (i) modelling the dynamic process of matte grade with the adjustment of operational parameter; (ii) optimizing the optimal operational parameters with related to expectative state by using model-based optimization method; and (iii) discretizing the differential and integral algebraic equations of eq. (1) to a NLP with PS method and solved it by a welldeveloped parameter optimization algorithm. Since solution (ii) beyond the scope of our discussion, detailed information may refer to literatures, we only discuss the method of (i) and (iii) in this paper.

\section{Solution Approach}

\subsection{Modelling dynamic process}

In practical, due to a number of energy transfer terms that are not well characterized or highly uncertain (e.g., radiation losses, energy involved in the combustion of the scrap material) about energy balance, ${ }^{28,29)}$ which makes it difficult to track the dynamics of temperature changes within the reaction shaft. In addition, the heat required, which is used to maintain the flash furnace temperature, is made up by combustion of heavy oil except for the generated heat from oxidation reaction. In addition, with the improving of the auto controlling application level in practical production plant, the furnace temperature can be controlled at a desired domain basically. Moreover, the characteristic of available measurement on-line for furnace temperature data makes it more sense to be utilized as a process variable for predicting matte grade. Thus, we assume the dynamic transient procedure as the isothermal process, and we also have relied on knowledge of the process and some simplifying assumptions reasonably according the actual copper smelting plant:

(i) assuming the copper concentrate is primarily $\mathrm{CuFeS}_{2}$ and $\mathrm{FeS}_{2}$, and the minerals $\mathrm{CuFeS}_{2}$ and $\mathrm{FeS}_{2}$ in the concentrate react rapidly and completely with seconds of being added to the furnace. ${ }^{30-32)}$

(ii) minor species such as $\mathrm{PbS}$ and $\mathrm{ZnS}$ in the matte and $\mathrm{Cu}_{2} \mathrm{O}, \mathrm{PbO}, \mathrm{ZnO}, \mathrm{CaO}$ and $\mathrm{Al}_{2} \mathrm{O}_{3}$ are not considered, and the $\mathrm{SiO}_{2}, \mathrm{~N}_{2}$ and $\mathrm{H}_{2} \mathrm{O}$ are chemically inert.

(iii) there is no accumulation of the gaseous species within the reaction shaft.

(iv) the species $\mathrm{SiO}_{2}, \mathrm{CaO}, \mathrm{Al}_{2} \mathrm{O}_{3}$ and $\mathrm{MgO}$ mostly exist in slag phase.

From the analysis previously, the reactions in the shaft of the furnace in essence can be viewed as the process of oxidative desulfurization and matte/slag forming. Studies have been completed to reveal the oxidative sulfur removal of copper concentrate as a function of time by using a shrinking-core model. ${ }^{4,5)}$

$$
1-(1-v)^{1 / 3}=k_{0} \exp \left(-\frac{E}{R T}\right) P_{\mathrm{O}_{2}}^{n} t
$$

where, $v$ is the removal ratio of sulfur $(\%), k_{0}$ is the preexponential factor, $E$ is the activation energy $(\mathrm{KJ} / \mathrm{mol}), R$ is the gas constant $(8.314 \mathrm{~J} /(\mathrm{mol} \cdot \mathrm{k}))$, and $T$ is the absolute temperature $(\mathrm{K}) . P_{\mathrm{O}_{2}}$ is the oxygen partial pressure $(\mathrm{KPa}), t$ is the reaction time (s) in the reaction shaft. The experience works have revealed that the reaction time $t$ of the particles in reaction shaft is almost decided by the structure of the furnace, so the time parameter $t$ can be considered as a pseudo-constant within the same furnace.

Due to the reactions take place at slight negative constant pressure, differential equations of oxygen partial pressure can be given by,

$$
\frac{\mathrm{d} P_{\mathrm{O}_{2}}}{\mathrm{~d} t}=\frac{\left[\left(\rho_{\mathrm{O}_{2}} \cdot u_{1}+0.217 \cdot u_{2}\right) * P_{\mathrm{T}}-\left(u_{1}+u_{2}\right) \cdot P_{\mathrm{O}_{2}}\right]}{V_{\mathrm{S}}} .
$$

In eq. (4), $\rho_{\mathrm{O}_{2}}$ is the efficient utilization coefficient of oxygen and is given by $\rho_{\mathrm{O}_{2}}=0.98$, approximately according to the actual smelting plant, $P_{\mathrm{T}}$ is the constant pressure and given by 

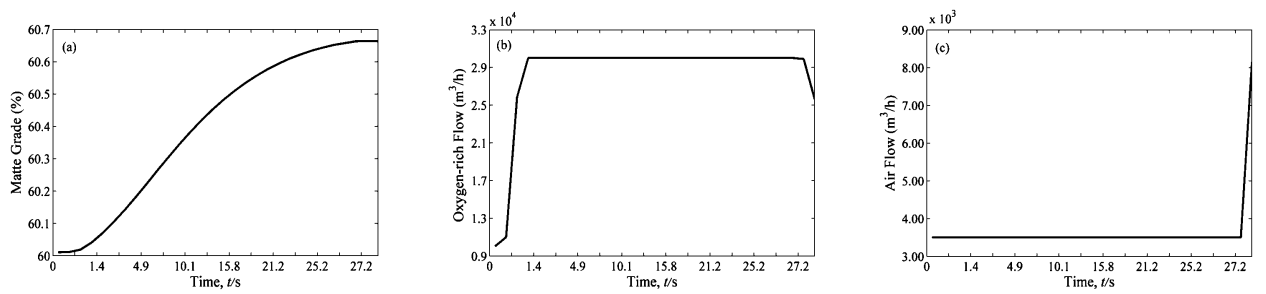

Fig. 1 Transient procedure of matte grade and the adjustment trajectories of oxygen-rich flow and air flow versus time with $\lambda=0$.
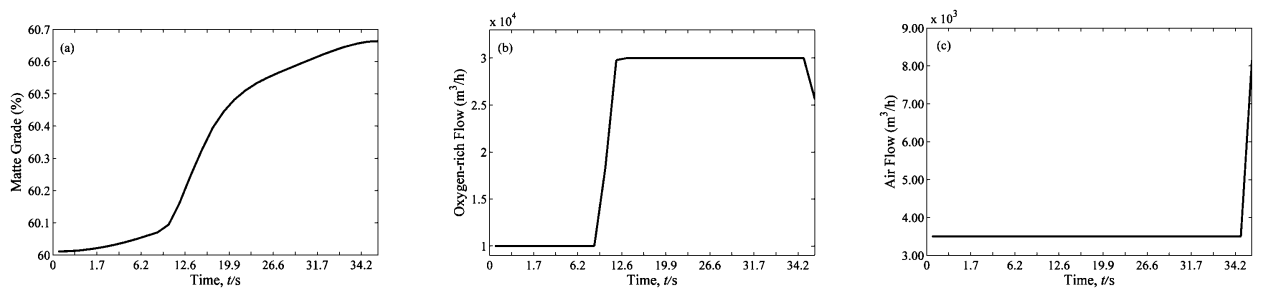

Fig. 2 Transient procedure of matte grade and the adjustment trajectories of oxygen-rich flow and air flow versus time with $\lambda=1$.

$P_{\mathrm{T}}=99.32(\mathrm{KPa})$ according to the actual production data, and $V_{\mathrm{S}}$ is the effective volume.

In addition, the formulation of copper losses in slag, ${ }^{33-36)}$ sulfur solubility capacity in the slag ${ }^{37}$ ) and the balance relationship of major components of $\mathrm{Cu}, \mathrm{Fe}$ and $\mathrm{S}$ are employed in the paper, the further details on them are described in the literature. ${ }^{11,14,18)}$

\subsection{Pseudospectral method}

To solve the transient cost-related optimization problem, a spectral algorithm known as the Legendre pseudospectral (PS) method is employed. For the purpose of clarity and brevity, we do not provide the Legendre PS method in detail, and the further details on the Legendre PS method can be viewed in the literatures. ${ }^{22,23}$ ) The method, available through a Matlab-based software package called DIDO, ${ }^{38)}$ discretizes the problem and approximates the state and control variables using Lagrange interpolating polynomials where the unknown coefficient values are obtained at the LegendreGauss-Lobatto (LGL) node points. The embedded nonlinear programming (NLP) solver SNOPT, ${ }^{39}$ ) which is based on sequential quadratic programming, then solves a sequence of finite-dimensional optimization problems that capture the full nonlinearities of the system.

\section{Results and Discussions}

This section is devoted to a numerical simulation experiment. We implement the transient cost-related optimization scheme proposed in this paper with DIDO software package within Matlab R2010a on a personal computer.

The ultimate goal of the transient cost-related optimization scheme proposed is to plan the optimal adjustment trajectories for operational parameters, which is used to ensure the dynamic transient procedure optimization. A sample (sampling time: 10:32 AM, 24th, Aug, 2006) is selected randomly as the initial parameters of the simulation in this section. For the reason of confidentiality, we cannot present the exact value for the sample data. The data demonstrate the excessive flow of $\mathrm{O}_{2}$ and slag in slag phase (copper mass fraction in slag) are $826 \mathrm{~m}^{3} / \mathrm{h}$ and $1.67 \%$, respectively, which more than normal losses about $533 \mathrm{~m}^{3} / \mathrm{h}$ (oxygen-flow calculated according to about $2 \%$ losses usually) and $0.9 \%$ (statistical value according to excellent conditions), respectively. So the utilization efficiency of copper concentrate and oxygen-rich can be improved further, and the corresponding working condition should be changed from the real-time state, $x\left(t_{0}\right)=60.01 \%$, to expectation, $x^{*}=60.66 \%$, which is optimized by using model-based optimization method, ${ }^{11,16)}$ and the related operational parameter of oxygen-rich and air flow also be optimized as $u_{1}^{*}=25620\left(\mathrm{~m}^{3} / \mathrm{h}\right)$ and $u_{2}^{*}=8135\left(\mathrm{~m}^{3} / \mathrm{h}\right)$, respectively. We applied the scheme proposed in the previous section to this problem with the assumption of isothermal during dynamic transient procedure, and the LGL points, $N=30$, is selected ${ }^{38)}$ meanwhile, only the flow of oxygen-rich is considered in the simulation example, namely, $\mu=1$. In addition, in order to reveal the impact degree of adjustment trajectory of operational parameters (the flow of oxygen-rich and air) on working state parameter (matte grade) transient procedure, different parameters values in transient costrelated optimization eq. (1) are considered, and the transient procedure of matte grade, the adjustment trajectories of the flows of oxygen-rich and air are given in Figs. 1-8 by using the proposed scheme in this paper.

Figures 1 and 2 demonstrate the transient procedure of matte grade with the adjustment trajectories of the oxygenrich and air flow, but without considering the fluctuation constraint of matte grade. From Fig. 1 and/or Fig. 2, the desired matte grade, $60.66 \%$, can be completed through $27.31 \mathrm{~s}$ without considering the minimization of oxygen-rich amount, and it also can be completed through $34.31 \mathrm{~s}$ with minimizing the oxygen-rich amount. The corresponding amounts of oxygen-rich of them are $224.77 \mathrm{~m}^{3}$ and $203.46 \mathrm{~m}^{3}$, respectively. The results of Figs. 1 and 2 shown that less time but more resources is consumed for the transition of matte grade from real-time state to expectation, and vice versa, which is consistent with the practical 

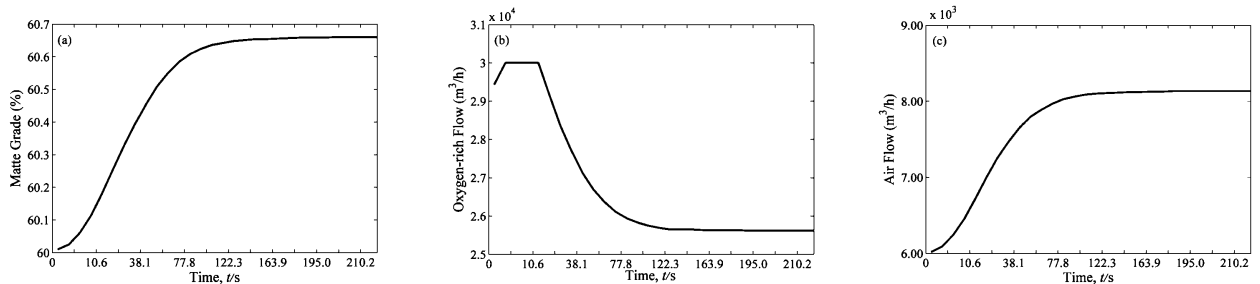

Fig. 3 Transient procedure of matte grade and the adjustment trajectories of oxygen-rich flow and air flow versus time with $\lambda=0$, $\sigma_{1}=0.5$ and $\sigma_{2}=0.5$.
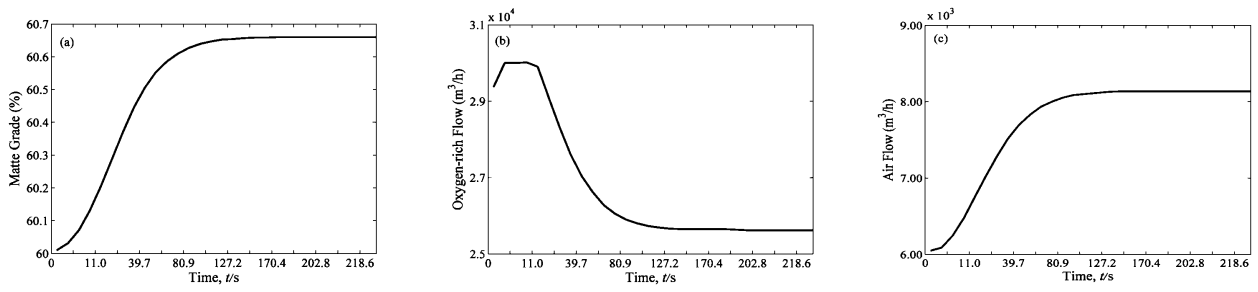

Fig. 4 Transient procedure of matte grade and the adjustment trajectories of oxygen-rich flow and air flow versus time with $\lambda=1$, $\sigma_{1}=0.5$ and $\sigma_{2}=0.5$.
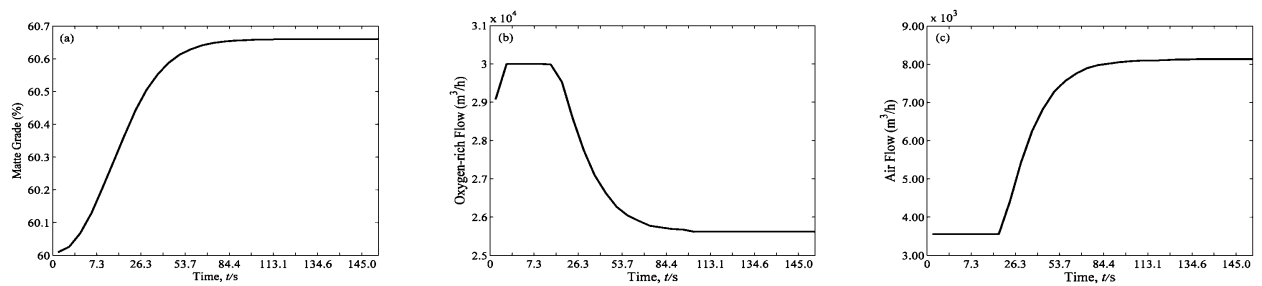

Fig. 5 Transient procedure of matte grade and the adjustment trajectories of oxygen-rich flow and air flow versus time with $\lambda=0$, $\sigma_{1}=0.8$ and $\sigma_{2}=0.2$
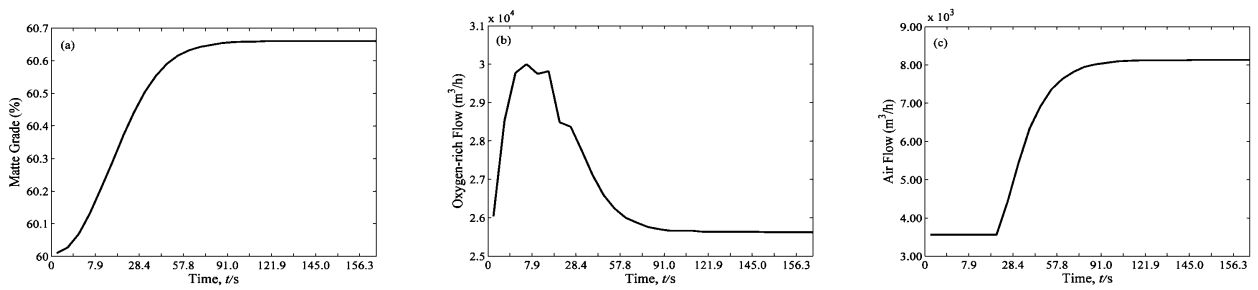

Fig. 6 Transient procedure of matte grade and the adjustment trajectories of oxygen-rich flow and air flow versus time with $\lambda=1$, $\sigma_{1}=0.8$ and $\sigma_{2}=0.2$.
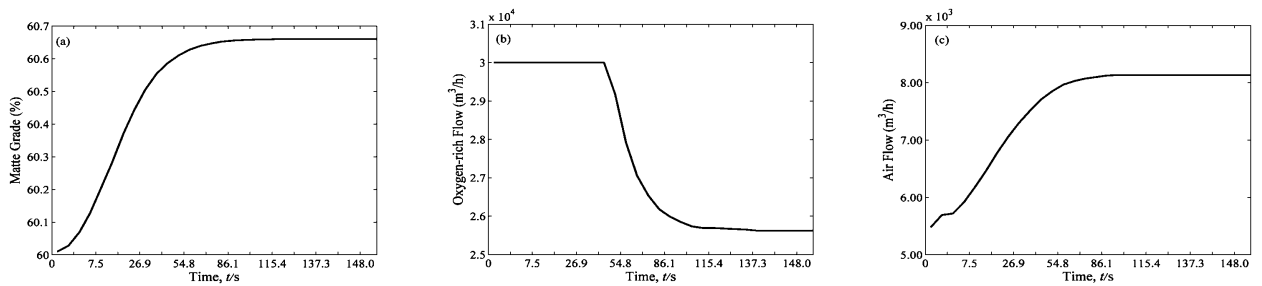

Fig. 7 Transient procedure of matte grade and the adjustment trajectories of oxygen-rich flow and air flow versus time with $\lambda=0$, $\sigma_{1}=0.2$ and $\sigma_{2}=0.8$.
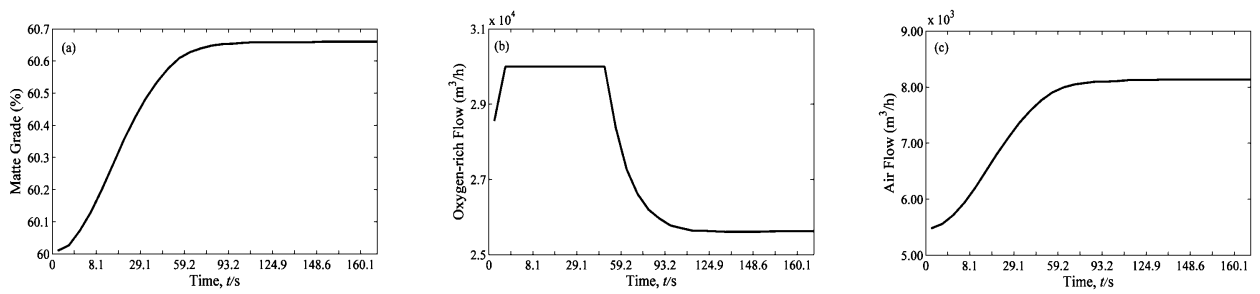

Fig. 8 Transient procedure of matte grade and the adjustment trajectories of oxygen-rich flow and air flow versus time with $\lambda=1$, $\sigma_{1}=0.2$ and $\sigma_{2}=0.8$. 
production process well. However, Figs. 1 and 2 also shown that the dramatic adjustment occurred near the steady-state point, which inevitable leads to dramatic fluctuation for matte grade, and these can be seen from the Figs. 1 and 2. In order to eliminate the dramatic fluctuation, we implement the fluctuation constraint eq. (2) in the scheme proposed in this paper and the corresponding results are given in Figs. 3-8.

From Figs. 1-8, the following several results can be devoted: (i) by comparison without considering minimizing resources indicator, the transient procedure can be achieved in less time with considering it; (ii) by comparison without fluctuation constraint, the transient procedure performs the smoother characteristic with considering it. Furthermore, with the deviation of real-time state from the expectation becomes smaller and smaller, the transient procedure of matte grade goes more and more smoother; (iii) the adjustment of oxygen-rich flow plays more important role on transient procedure of matte grade than if of air flow.

Practically, the ultimate goal of solving transient costrelated optimization problem for copper flash smelting process is to ensure the dynamic transient procedure can be received within the least time while as little resources consumption as possible, as well as the smooth behaviors. Moreover, the consumption of oxygen-rich amount during the dynamic transient procedure is the main concern for resources consumption. Thus, the model parameters $\lambda$ and $\mu$ can be chosen as $\lambda=1$ and $\mu=1$, respectively. Meanwhile, in order to ensure the smoothness behavior during the dynamic transient procedure, the parameter with stronger effect on performance index (matte grade) should be adjusted in advance, which makes the adjustment amplitude smoother near by the steady-state point. So, the model parameter in eq. (2) can be set as $\sigma_{1}=0.8$.

\section{Conclusion}

In the present work, in order to solve the optimization transition problems for copper flash smelting process, combined with the actual copper flash smelting process at a Smelter in China, a transient cost-related optimization problem was formulated in advance. Then it was described as an operational parameters trajectories optimization problem, where subject to the equality and inequality hybrid constraints. Meanwhile, by limiting substantial adjustment for operational parameters, a fluctuation constraint was presented to ensure the smoothness of state during dynamic transient procedure. In addition, the PS-based optimization software package DIDO was employed to solve the transient cost-related optimization problem.

The numerical simulation results and discussions have shown that the optimal operational parameters adjustment trajectories can be constructed with relation to the corresponding performance index, which is useful in providing guidance for the adjustment of operational parameters for actual production process. They also revealed the opposition characteristic of transient time to transient resources consumption. In addition, by comparison with the adjustment of air flow, the adjustment of oxygen-rich flow plays a more important role in changing matte grade. The next main conclusion is that the fluctuation constraint employed can eliminate effectively the dramatic fluctuation of working state parameter.

The next step in our work is to implement the proposed transient cost-related optimization scheme on the actual copper flash smelting process manufactured by a Smelter in China. And we believe that the proposed scheme can reduce resources consumption and improve production efficiency, bringing about huge economic benefits for company.

\section{Acknowledgements}

The authors of this work acknowledge the support of the National Funds for Distinguished Young Scientists of China (61025015), and the National Natural Science Foundation of China (61074177) over the duration of this project.

\section{REFERENCES}

1) D. R. Higgins, N. B. Gray and M. R. Davidson: Miner. Eng. 22 (2009) 1251-1265.

2) A. H. Alyaser and J. K. Brimacombe: Metall. Mater. Trans. B 26 (1995) 25-40.

3) X. F. Li, S. H. Peng, X. L. Han, C. Mei and T. Y. Xiao: J. Univ. Sci. Technol. B 11 (2004) 115-119.

4) B. S. Kim, H. I. Lee, J. T. Park, J. S. Sohn and J. C. Lee: Mater. Trans. 49 (2008) 1889-1892.

5) B. S. Kim, E. Y. Kim, C. K. Kim, H. I. Lee and J. S. Sohn: Mater. Trans. 49 (2008) 1192-1198.

6) I. Harjunkoski, H. W. Borchers and M. Fahl: Comput-Aided Chem. Eng. 21 (2006) 1197-1202.

7) M. Karrari, W. Rosehart and O. P. Malik: Asian J. Control. 7 (2005) 286-295.

8) T. Norgate and S. Jahanshahi: Miner. Eng. 23 (2010) 65-73.

9) F. Bakhtiari, H. Atashi, M. Zivdar, S. Seyedbagheri and M. H. Fazaelipoor: J. Ind. Eng. Chem. 17 (2011) 29-35.

10) I. Jovanović, P. Stanimirović and Ž. Živković: Environ. Model. Assess. 18 (2013) 73-83.

11) X. B. Peng, W. H. Gui, Y. G. Li, Z. K. Hu and L. Y. Wang: Proc. 2007 IEEE Conf. Cont. Autom., (ICCA, Guang-zhou, China, May 2007) 2328-2333.

12) L. Pradenas, J. Zúñiga and V. Parada: Interfaces 36 (2006) 296-301.

13) M. Schaaf, Z. Gómez and A. Ciprianom: J. Process Contr. 20 (2010) 3-17.

14) W. H. Gui, L. Y. Wang, C. H. Yang, Y. F. Xie and X. B. Peng: T. Nonferr. Metal. Soc. China 17 (2007) 1075-1081.

15) X. F. Li, C. Mei, P. Zhou, X. L. Han and T. Y. Xiao: T. Nonferr. Metal. Soc. China 13 (2003) 203-207.

16) H. R. Chen, C. Mei, K. Xie, X. F. Li, J. Zhou, X. H. Wang and Z. L. Ge: T. Nonferr. Metal. Soc. China 14 (2004) 631-636.

17) S. L. Jämsä-Jounela, M. Vermasvuori, P. Endén and S. Haavisto: Control Eng. Pract. 11 (2003) 83-92.

18) R. Parada, R. Parra and I. Wilkomirsky: Can. Metall. Quart. 43 (2004) 561-570.

19) Z. Z. Zhu and J. Q. He: The Modern Metallurgy, (Science Press, Beijing, 2003) (in Chinese).

20) M. Shamsi: Optim. Contr. Appl. Met. 32 (2011) 668-680.

21) H. P. Ma, T. H. Qin and W. Zhang: IEEE T. Automat. Contr. 56 (2011) $675-680$.

22) G. Qi, K. Wei and I. M. Ross: IEEE T. Automat. Contr. 51 (2006) 1115-1129.

23) G. Elnagar, M. A. Kazemi and M. Razzaghi: IEEE T. Automat. Contr. 40 (1995) 1793-1796.

24) R. J. Vanderbei: Optim. Eng. 2 (2001) 215-243.

25) D. Garg, W. W. Hager and A. V. Rao: Automatica 47 (2011) 829-837.

26) J. Nocedal and S. t. Wright: Numerical Optimization, 2nd ed., (Springer Series in Operations Research, Springer, New York, 1999).

27) M. Wächter and G. Sachs: Asian J. Control. 8 (2006) 307-313.

28) J. W. Matousek: JOM-J. Min. Met. Mat. S. 61 (2009) 61-63. 
29) R. Sridhar, J. M. Toguri and S. Simeonov: JOM-J. Min. Met. Mat. S. 49 (1997) 48-52.

30) P. Ronan, M. Pritzker and H. M. Budman: Ind. Eng. Chem. Res. 36 (1997) 112-121.

31) W. Davenport and E. Partelpoeg: Flash Smelting, Analysis, Control and Optimization, (Pergamon Press, Oxford, 1987).

32) S. L. Cain, H. M. Budman and M. Pritzker: Can. J. Chem. Eng. 74 (1996) 993-1003.

33) W. G. Davenport, M. King, M. Schlesinger and A. K. Biswas: Extractive Metallurgy of Copper, 4th ed., (Pergamon Press, New York, 2002).

34) P. Shimpo, S. Goto, O. Ogawa and I. Asakura: Can. Metall. Quart. 25
(1986) 113-121.

35) Ž. Živković, N. B. Mitevska, I. Mihajlović and Đ. Nikolić: Miner. Metall. Proc. 27 (2010) 141-147.

36) Ž. Živković, N. B. Mitevska, I. Mihajlović and Đ. Nikolić: J. Min. Metall. B 45 (2009) 23-34.

37) M. Nagamori: Metall. Trans. 5 (1974) 531-538.

38) I. M. Ross: A beginner's guider to DIDO: A Matlab application package for solving optimal control problem. Elissar Technical Report TR-711, http://www.elissar.ziz, 2007.

39) P. E. Gill, W. Murray and M. A. Saunders: User's guide for SNOPT Version 7: Software for large-scale nonlinear programming. Technical Report, Stanford Business Software, Jun, 2008. 